\title{
Thermo-Coulomb effects in mesoscopic tunnel junctions
}

\author{
M. Amman', E. Ben-Jacob' ${ }^{2}$, and J. Cohn ${ }^{3}$ \\ ${ }^{1}$ Department of Physics, University of Michigan, Ann Arbor, MI 48109, USA \\ ${ }^{2}$ Raymond and Beverly Sackler, Faculty of Exact Sciences, School of Physics and Astronomy, Tel Aviv University, 69978 Tel Aviv, Israel \\ ${ }^{3}$ Naval Research Laboratories, Washington, DC 20375, USA
}

Received June 25, 1991; revised version September 9, 1991

We present a study of the thermoelectric effects in tunnel junctions. In particular we calculate the thermopower coefficient $S$ and the Peltier coefficient $\Pi$. For macroscopic junctions we demonstrate the sensitivity of $S$ and $\Pi$ to the structure of the density of states. For mesoscopic junctions we show that Coulomb effects modify the ordinary Onsager picture and the relation $\Pi=T S$. The coefficients $S$ and $\Pi$ are found to be very sensitive to the coupling of the junctions to the external world. We analyze measurements of the thermopower in granular films in terms of the thermo-Coulomb effects. We comment on the relevance of these effects to scanning tunneling microscope measurements.

\section{Introduction}

During the last five years, considerable interest has been directed towards tunnel junction systems where the discreteness of the electronic charge plays a prominent and observable role [1]. These systems involve at least one ultra-small conductive element with a capacitance $C$, such that its charging energy $E_{c}$ is larger than the thermal energy:

$E_{c} \equiv e^{2} / 2 C \gg k_{B} T$.

The theoretical and experimental study spans from the simplest element - a single mesoscopic normal tunnel junction - to systems of 2-D arrays of junctions. New phenomena, such as the Coulomb blockade, Coulomb staircase, and single charge solitons have been predicted and observed. All these phenomena depend on the electric response of the systems, that is, the response to an external driving voltage, charge, or current sources.

Being mesoscopic systems, their electric response is crucially sensitive to the nature of the external driving circuit. Motivated by the new understanding, we expect that the thermal response of the junctions will show phe- nomena analogous to those studied in the electrical response, while new phenomena will be observed in the thermoelectric response [2].

As a first step in this direction we present here a study of two thermoelectric effects, Seebeck and Peltier, in mesoscopic normal junctions. We show that the temperature dependence of the thermopower coefficient $S$ and the Peltier coefficient $\Pi$ is very sensitive to the nature of the external circuit. Moreover, the ordinary Onsager picture [3] has to be modified as well as the relation $\Pi=T S$.

In Sect. 2, we review the definition of $S$ and $\Pi$. We demonstrate the sensitivity of these coefficients to the structure of the normalized density of states in Sect. 3. We show that, for a finite energy gap, $S$ diverges as $1 / T$, while for a dip in the density of states (DOS) it decreases exponentially with decreasing temperature. Our main message concerns thermo-Coulomb effects - thermoelectric effects in mesoscopic junctions where charging effects are important. We demonstrate the sensitivity of $S$ and $\Pi$ to the coupling of the junctions to the external circuit (measuring or driving) and discuss the validity of the Onsager picture in such junctions, in Sect. 4.

In Sect. 5, we analyze measurements of the thermopower in a granular material in terms of thermo-Coulomb effects. Finally, the conclusions are presented in Sect. 6.

\section{The thermopower and the Peltier coefficients}

The Seebeck effect is the development of a voltage $V$ across an open junction (zero electric current) when a temperature gradient $\Delta T$ is applied. The operational definition of the thermopower coefficent $S$ is

$$
S \equiv \lim _{\Delta T \rightarrow 0} \frac{V}{\Delta T},
$$

where $V$ is the measured voltage across the open junction (infinite external resistance).

Alternatively, $V$ is defined as the applied voltage required to balance the current due to the temperature gra- 
dient. For macroscopic junctions both definitions yield the same result. Moreover, within linear response theory (applicable for macroscopic tunnel junctions), the electric current passed through the junction is

$$
\begin{aligned}
I(V, \Delta T) \approx & \left.\lim _{\Delta T \rightarrow 0} \frac{\partial I}{\partial \Delta T}\right|_{V=0} \Delta T \\
& +\left.\lim _{V \rightarrow 0} \frac{\partial I}{\partial V}\right|_{\Delta T=0} V .
\end{aligned}
$$

Hence, the thermopower coefficient for $I(V, \Delta T)=0$ may be expressed as

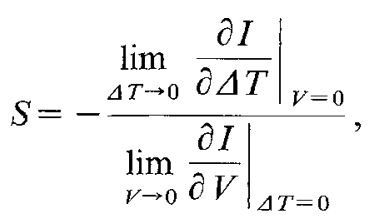

which is the form typically used for calculations.

The Peltier effect refers to the heat current that passes across the junction when the two sides are kept at equal temperature and an electric current $I$ is forced to flow. Accordingly, the Peltier coefficient $\Pi$ is defined as

$\left.\Pi \equiv \lim _{I \rightarrow 0} \frac{\partial I_{Q}}{\partial I}\right|_{\Delta T=0}$.

Within linear response theory, using the relation $\frac{\partial I}{\partial V}=\left[\frac{\partial V}{\partial I}\right]^{-1}, \Pi$ is given by

$\Pi=\frac{\left.\lim _{V \rightarrow 0} \frac{\partial I_{Q}}{\partial V}\right|_{\Delta T=0}}{\left.\lim _{V \rightarrow 0} \frac{\partial I}{\partial V}\right|_{\Delta T=0}}$

which has a form equivalent to that of (4). Using the Onsager relations for the off-diagonal terms

$\left.\frac{\partial I_{Q}}{\partial V}\right|_{\Delta T=0}=\left.T \frac{\partial I}{\partial \Delta T}\right|_{V=0}$,

we obtain the following relation

$\Pi=T S$.

Note that, similarly to (5), we can obtain

$S=\left.\lim _{I \rightarrow 0} \frac{\partial I_{S}}{\partial I}\right|_{\Delta T=0}$,

which motivates us to view $S$ as the measure of the entropy transferred per carrier.

\section{The effect of the normal density of states}

In this section we consider macroscopic junctions, so that the charging energy contribution may be ignored and the linear response definitions of the thermopower coefficient and the Peltier coefficient are applicable. For such a tunnel junction, the electric current passed through it is $I=e(r-l)$, where the electron tunneling rates from the right and left, respectively, are

$$
\begin{aligned}
r= & \frac{1}{e^{2} R} \int_{-\infty}^{+\infty} D_{l}(E) D_{r}(E-e V) f^{T}(E-e V) \\
& \times\left[1-f^{T+\Delta T}(E)\right] \mathrm{d} E, \\
l= & \frac{1}{\mathrm{e}^{2} R} \int_{-\infty}^{+\infty} D_{l}(E) D_{r}(E-e V) f^{T+\Delta T}(E) \\
& \times\left[1-f^{T}(E-e V)\right] \mathrm{d} E .
\end{aligned}
$$

Here $f^{T}(E)=1 /\left[\exp \left(E / k_{B} T\right)+1\right]$ is the Fermi-Dirac distribution function, $D_{r}(E)$ and $D_{l}(E)$ are the right and left electrode normalized DOS [4], and $R$ is the normal state resistance of the junction, which includes the normalization factors of $D_{r}(E)$ and $D_{l}(E)$.

The partial derivatives of the current with respect to the temperature difference and the voltage are given by

$$
\begin{aligned}
& \left.\lim _{\Delta T \rightarrow 0} \frac{\partial I}{\partial \Delta T}\right|_{V=0} \\
& =\frac{1}{e R} \int_{-\infty}^{+\infty} D_{l}(E) D_{r}(E) \frac{E}{T} \frac{\partial f^{T}(E)}{\partial E} \mathrm{~d} E, \\
& \left.\lim _{\Delta T \rightarrow 0} \frac{\partial I}{\partial V}\right|_{\Delta T=0} \\
& =\frac{1}{R} \int_{-\infty}^{+\infty} D_{l}(E) D_{r}(E)\left(-\frac{\partial f^{T}(E)}{\partial E}\right) \mathrm{d} E .
\end{aligned}
$$

For normal metal tunnel junctions we evaluate the integrals using the Sommerfeld expansion

$\int_{-\infty}^{+\infty} G(E)\left[-\frac{\partial f^{T}(E)}{\partial \mathrm{E}}\right] \mathrm{d} E \approx G(0)+\frac{\pi}{6}\left(k_{B} T\right)^{2} G^{\prime \prime}(0)$

and obtain

$\left.\lim _{\Delta T \rightarrow 0} \frac{\partial I}{\partial \Delta T}\right|_{V=0} \approx-\frac{\pi^{2} k_{B}^{2} T}{3 e R}\left[D_{l}(0) D_{r}(0)\right]^{\prime}$,

$$
\begin{aligned}
& \left.\lim _{V \rightarrow 0} \frac{\partial I}{\partial V}\right|_{\Delta T=0} \\
& \quad \approx \frac{1}{R}\left\{D_{l}(0)_{r}(0)+\frac{1}{6}\left(\pi k_{B} T\right)^{2}\left[D_{l}(0) D_{r}(0)\right]^{\prime \prime}\right\} .
\end{aligned}
$$

The thermopower coefficient is then given by

$$
S=\frac{\pi^{2} k_{B}^{2} T}{3 e} \frac{\left[D_{l}(0) D_{r}(0)\right]^{\prime}}{D_{l}(0) D_{r}(0)+\frac{1}{6}\left(\pi k_{B} T\right)^{2}\left[D_{l}(0) D_{r}(0)\right]^{\prime \prime}} .
$$


Note that, when the second derivative may be ignored, $S$ is proportional to the temperature times the logarithmic derivative of the normalized DOS, which is the known result for bulk metals.

Next, we consider a model junction (motivated by the semiconductor DOS) with electrodes that have the following normalized DOS

$D(E)=\left\{\begin{array}{lc}D_{b} & E<-\Delta \\ 0 & -\Delta<E<\Delta \\ D_{a} & E>\Delta\end{array}\right.$

where $D_{b}$ and $D_{a}$ are constant normalized DOS below and above the gap of size $2 \Delta$. Since there is a finite gap, the Sommerfeld expansion is not applicable. To proceed, we express the current as

$$
\begin{aligned}
I= & \frac{D_{a}^{2}}{e R} \int_{e V+\Delta}^{+\infty}\left[f^{T}(E-e V)-f^{T+\Delta T}(E)\right] \mathrm{d} E \\
& +\frac{D_{b}^{2}}{e R} \int_{-\infty}^{-\Delta}\left[f^{T}(E-e V)-f^{T+\Delta T}(E)\right] \mathrm{d} E .
\end{aligned}
$$

For low temperatures, $\Delta \gg k_{B} T$, the Fermi functions of the first integral may be approximated as $f(E) \approx \exp \left(-E / k_{B} T\right)$ and one minus the Fermi functions of the second integral can be approximated as $1-f(E) \approx \exp \left(E / k_{B} T\right)$. Using these approximations, (19) is integrated and

$$
\begin{aligned}
I \approx & \frac{D_{a}^{2}}{e R}\left[k_{B} T \exp \left(-\frac{\Delta}{k_{B} T}\right)\right. \\
& \left.-k_{B}(T+\Delta T) \exp \left(-\frac{e V+\Delta}{k_{B}(T+\Delta T)}\right)\right] \\
& +\frac{D_{b}^{2}}{e R}\left[k_{B}(T+\Delta T) \exp \left(-\frac{\Delta}{k_{B}(T+\Delta T)}\right)\right. \\
& \left.-k_{B} T \exp \left(-\frac{e V+\Delta}{k_{B} T}\right)\right] .
\end{aligned}
$$

The thermoelectric power may now be calculated from (3) to give

$$
\begin{aligned}
S & =\frac{\left(k_{B}+\frac{\Delta}{T}\right)\left(D_{b}^{2}-D_{a}^{2}\right) \exp \left(-\frac{\Delta}{k_{b} T}\right)}{e\left(D_{b}^{2}+D_{a}^{2}\right) \exp \left(-\frac{\Delta}{k_{B} T}\right)} \\
& \approx \frac{k_{B}}{e}\left(\frac{D_{a}^{2}-D_{b}^{2}}{D_{a}^{2}+D_{b}^{2}}\right) \frac{\Delta}{k_{B} T} .
\end{aligned}
$$

In this case, both the electric conductivity $\left(\frac{\partial I}{\partial V}\right)$ and the temperature gradient contribution to the current $\left(\frac{\partial I}{\partial \Delta T}\right)$ are exponentially small with the same exponen tial dependence $\left(\propto \exp \left(-\frac{\Delta}{k_{B} T}\right)\right)$. Therefore, $S$ is proportional to the ratio of the prefactors. This result is qualitatively different if there are allowed energy states in the gap region. For example, if there is a small density of states in the gap region, the numerator of (21) will still

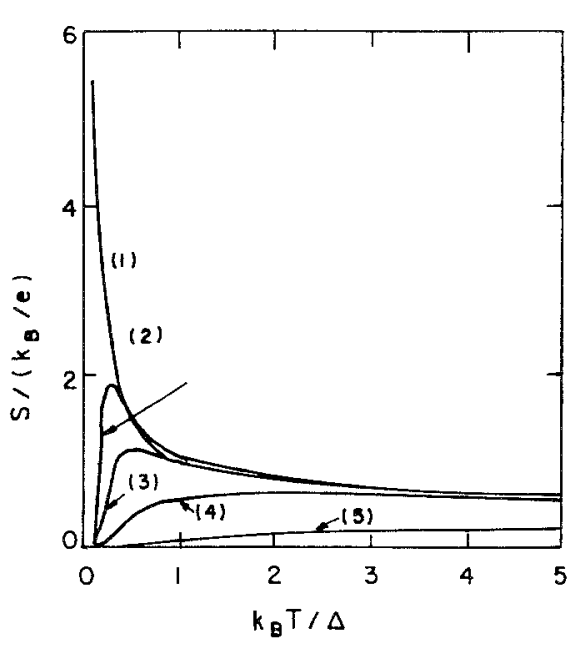

Fig. 1. The effect of gap states $(D g)$ on the thermopower. Equation (22) for $D_{a} / D_{b}=1.5$. The lines (1)-(5) are for $D_{g} / D_{b}=0.0,0.01$, $0.1,1.0,10.0$ respectively

be exponentially small for low temperatures, but the denominator will contain algebraic terms in $1 / T$. Hence, the exponential terms will no longer cancel and $S$ will exponentially decrease as $T \rightarrow 0$. For a finite density of states in the gap, such that

$D(E)=\left\{\begin{array}{lc}D_{b} & E<-\Delta \\ D_{g} & -\Delta<E<\Delta, \\ D_{a} & E>\Delta\end{array}\right.$

the thermopower coefficient is

$$
\begin{aligned}
\frac{S}{k_{B} / e}= & \frac{\Delta}{k B} T \\
& \times\left[1+\frac{k_{B} T}{\Delta}\left(\exp \frac{\Delta}{k_{B} T}+1\right)\right. \\
& \left.\times \ln \left(\exp -\frac{\Delta}{k_{B} T}+1\right)\right] \\
& \times \frac{\left[D_{a}^{2}-D_{b}^{2}\right]}{D_{a}^{2}+D_{b}^{2}+D_{g}^{2}\left(\exp \frac{\Delta}{k_{B} T}-1\right)}
\end{aligned}
$$

In Fig. 1 we show the effect of $D_{g}$ on the temperature dependence of $S$.

\section{Thermo-Coulomb effects in mesoscopic normal tunnel junctions}

The response of a mesoscopic tunnel junction strongly depends on its coupling to the "external world" (the impedance of the external driving or measuring circuit). For example, the $I-V$ characteristic of a current driven junction is qualitatively different from that of a voltage biased junction. Consequently, one must reinterpret the standard Onsager picture

$$
\begin{aligned}
& \mathbf{J}=[L] \mathbf{F}, \\
& L_{12}=L_{21}
\end{aligned}
$$


where $J$ are the generalized fluxes and $F$ are the generalized forces. For the thermoelectric effects

$$
\begin{aligned}
& J_{1}=\frac{I}{e}, \quad F_{1}=\frac{1}{T} \Delta \mu=\frac{e V}{T} \\
& J_{2}=I_{Q}, \quad F_{2}=\Delta\left(\frac{1}{T}\right) .
\end{aligned}
$$

This picture holds only in the limit of "forces control", that is, voltage and temperature gradient $\Delta T$ bias in the thermoelectric case. We emphasize that both the thermopower and Peltier coefficients are originally defined for current control. (An open junction may be viewed as current controlled with $I=0$.) Hence, for mesoscopic junctions, there is no unique definition for $S$ and $\Pi$, and each possible definition (which yields a different temperature dependence of the coefficients) depends on the measurement setup.

We proceed now to calculate the thermopower coefficient for three limits. First, following the original definition for $S$, we obtain

$S \equiv \frac{\langle V\rangle_{\text {time }}}{\Delta T}$

Here $\langle V\rangle_{\text {time }}$ is the time averaged voltage of an open circuit junction. This case is equivalent to a zero current source, that corresponds to the limit

$\tau_{T}<\tau_{D}<\tau_{R}$,

where $\tau_{T}$ is the time of the tunneling [5], $\tau_{D}$ is the dwell time (the time between tunneling events) and $\tau_{R}$ is the response of the external circuit (infinite in this case). The average voltage is given by

$\frac{C\langle V\rangle_{\mathrm{time}}}{e}=\langle n\rangle=\sum_{n=-\infty}^{\infty} n \rho_{\mathrm{eq}}(n)$

where $\langle n\rangle$ is the average number of charges that have tunneled across the junction (in the direction of the temperature gradient). $\rho_{\mathrm{eq}}(n)$ is the steady state solution of the master equation [6]

$$
\begin{aligned}
\frac{\partial \rho(n, t)}{\partial \mathrm{t}}= & r(n+1) \rho(n+1, t)+l(n-1) \rho(n-1, t) \\
& -[r(n)+l(n)] \rho(n, t),
\end{aligned}
$$

where the electron tunneling rates are

$$
\begin{aligned}
r(n)= & \frac{1}{e^{2} R} \int_{-\infty}^{+\infty} D_{l}\left(E-\frac{e^{2}}{2 C}\right) \\
& \times D_{r}\left(E-\frac{n e^{2}}{C}\right) f^{T}\left(E-\frac{n e^{2}}{C}\right) \\
& \times\left[1-f^{T+\Delta T}\left(E-\frac{e^{2}}{2 C}\right)\right] \mathrm{d} E
\end{aligned}
$$

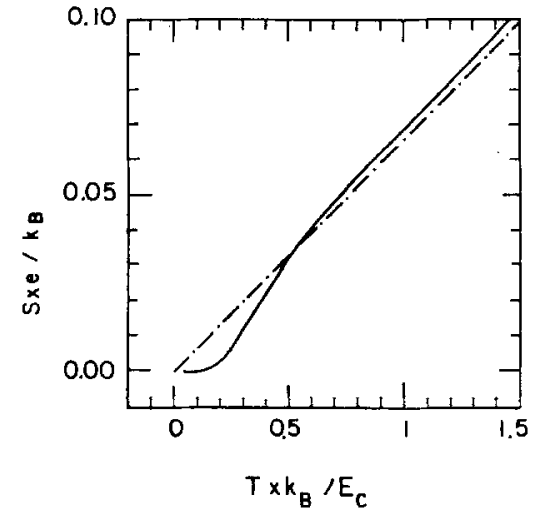

Fig. 2. Solid line: Calculated thermopower for a mesoscopic open junction. The electrode normalized density of states are assumed to be linear $D(E)=D_{1}+D_{2} E / E_{c}$, with $D_{2} / D_{1}=0.01$. The calculation is made for a fixed ratio $\Delta T / T=0.01$. Dashed line: Calculated thermopower for the same system as the solid line except the tunneling rates used in the calculation do not include the charging energy

$$
\begin{aligned}
l(n)= & \frac{1}{e^{2} R} \int_{-\infty}^{+\infty} D_{l}(E) D_{r}\left(E-\frac{n e^{2}}{C}-\frac{e^{2}}{2 C}\right) \\
& \times f^{T+\Delta T}(E)\left[1-f^{T}\left(E-\frac{n e^{2}}{C}-\frac{e^{2}}{2 C}\right)\right] \mathrm{d} E
\end{aligned}
$$

Following Amman et al. [5], $\rho_{\text {eq }}(n)$ is given by

$$
\rho_{\mathrm{eq}}(n)=\frac{\left[\prod_{i=-\infty}^{n-1} l(i)\right]\left[\prod_{i=n+1}^{+\infty} r(i)\right]}{\sum_{j=-\infty}^{+\infty}\left[\prod_{i=-\infty}^{j-1} l(i)\right]\left[\prod_{i=j+1}^{+\infty} r(i)\right]} .
$$

In this limit, the charging energy reduces the tunneling rates, and hence $S$ decreases exponentially at low temperatures, as shown in Fig. 2.

The thermopower may be interpreted as the imposed voltage required for $I=0$ when a temperature gradient $\Delta T$ is applied. When an ideal voltage source is assumed ( $\tau_{R}$ is smaller than $\tau_{T}$ and $\tau_{D}$, or zero external impedance), the external circuit shorts the capacitance, and $S$ is the same as for a macroscopic junction [Eq. (17)].

A more interesting case is that of a dwell source (intermediate external impedance), that is

$\tau_{T}<\tau_{R}<\tau_{D}$

This limit may be either a closed circuit junction with a non-ideal voltage source or an open circuit with a nonideal voltmeter. To calculate the thermopower coefficient we use the definition of $S$ in (4), $I=e(r-l)$, and the transition rates

$$
\begin{aligned}
r= & \frac{1}{e^{2} R} \int_{+\infty}^{-\infty} D_{l}\left(E-E_{c}\right) D_{r}(E-e V) f^{T}(E-e V) \\
& \times\left[1-f^{T+\Delta T}\left(E-E_{c}\right)\right] \mathrm{d} E .
\end{aligned}
$$


( $l$ is similarly defined.) In the limit $E_{c} \ll k_{B} T, S$ is given by (17). In the opposite limit we approximate $f(1-f)$ in the equation for $r$ as a constant $h$ in the range $e V<E<E_{c}$

$h \approx \exp \left(-\frac{E-e V}{k_{B} T}\right) \exp \left(\frac{E-E_{c}}{k_{B}(T+\Delta T)}\right)$.

Using this approximation and a similar one for $l$ we get

$S \approx \frac{k_{B}}{e} D\left(E_{c}\right) \frac{E_{c}}{k_{B} T}$,

where

$$
=\frac{\int_{0}^{E_{c}}\left[D_{l}\left(E-E_{c}\right) D_{r}(E)\left(\frac{E}{E_{c}}-1\right)+D_{l}(E) D_{r}\left(E-E_{c}\right) \frac{E}{E_{c}}\right] \mathrm{d} E}{\int_{0}^{E_{c}}\left[D_{l}\left(E-E_{c}\right) D_{r}(E)+D_{l}(E) D_{r}\left(E-E_{c}\right)\right] \mathrm{d} E} .
$$

For macroscopic junctions the Peltier coefficient $\Pi$ is directly obtained from $S$, using (8). We emphasize again that $\Pi$ is the average energy carried by the tunneling charges. Hence, the heat current $I_{Q}$ differs from the Joule heat which is a measure of the heat production rate (due to the current $I$ ).

For mesoscopic junctions (8) does not hold, and $\Pi$ has to be derived separately. The original definition of the Peltier effect implicitly assumes the current source limit. In this limit, the entropy productin due to the charge tunneling (in the direction of the voltage drop) is:

$\Delta S=\frac{\left(e V_{T}-\frac{e^{2}}{2 C}\right)}{T}$,

where $V_{T}$ is the voltage before the tunneling event. Hence, $\langle\Delta S\rangle=\frac{e\langle\Delta V\rangle}{T}$ and the Joule heating is $I\langle\Delta V\rangle$ (as expected).

The averaged excess energy transferred in each of the tunneling events is $\left(e V_{T}+\frac{e^{2}}{2 C}\right)$. Therefore, the averaged
heat current is

$$
\begin{aligned}
\left\langle I_{Q}\right\rangle & =\frac{1}{2}\left(\left\langle V_{T}\right\rangle+\frac{e}{2 C}\right) I \\
& =\frac{1}{2}\left(\langle V\rangle+\frac{e}{C}\right) I .
\end{aligned}
$$

A more detailed discussion of the time dependence will be given in a forthcoming publication.

\section{Thermopower and magneto-resistance of oxidized bismuth films}

In this section we review low-temperature measurements of resistance, magnetoresistance, and thermoelectric

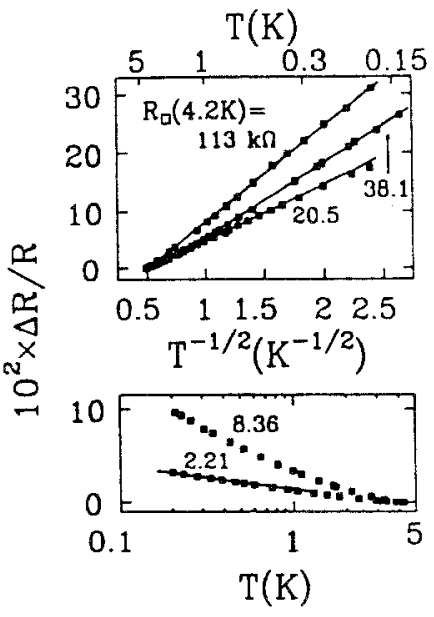

Fig. 3. Relative resistance increase, $[R(T)-R(4.2 \mathrm{~K})] / R(4.2 \mathrm{~K})$, versus temperature for a single $\mathrm{Bi}$ film oxidized in steps. Each curve is labeled by the value of $R(4.2 \mathrm{~K})$

power in semicontinuous bismuth films [7]. The coupling between grains is controlled by sequentially oxidizing individual films. Our focus is on the crossover regime where we observe a new and striking behavior of the thermopower. A possible explanation based on the Coulomb blockade in small-capacitance tunnel junctions is proposed. Magnetoresistance data suggest that a sharp charging energy onset occurs when the average intergrain tunnel resistance exceeds $\sim \hbar / e^{2}$.

Samples were prepared by vapor deposition of $99.9999 \%$ purity $\mathrm{Bi}$ onto room temperature glass substrates in an oxygen atmosphere of 50 mTorr. The films were deposited in a four-probe bridge pattern $\left(0.8 \times 10 \mathrm{~mm}^{2}\right)$ with nominal thickness $55-70 \AA$ as determined by a quartz crystal monitor. A $300 \AA$ SiO layer was then deposited over the conduction channel, allowing for slower, controllable oxidation. Films prepared in this fashion had post-deposition (dc) sheet resistances, $R$, of $<1 \mathrm{k} \Omega$. Transmission electron and scanning tunneling microscopies indicate that the films are composed of closely packed crystalline Bi grains which form a multiply connected, filamentary structure. The grains are irregularly shaped with diameters ranging from 200 to $1000 \AA$. Exposing the films to air at room temperature promotes the growth of insulating $\mathrm{Bi}_{2} \mathrm{O}_{3}$ at the granular surfaces. The corresponding increases in $R$ to values $>100 \mathrm{k} \Omega$ are associated with a reduced intergrain coupling and the development of a percolative structure, as discussed in detail elsewhere. Details of the experiments are described in [7].

Figure 3 shows the typical low-temperature resistance behavior for one of our films. For $R(4.2 \mathrm{~K}) \ll 8 \mathrm{k} \Omega$ the resistance increases as $\ln T$, with a slope that agrees with weak disorder theories for two-dimensional systems [8] and is consistent with previous studies of thin, homogeneous Bi films. The temperature dependence for $R(4.3 \mathrm{~K})$ $>8 \mathrm{k} \Omega$ is faster than $\ln T$ (approximately $T^{-1 / 2}$ ), but not yet exponential. For example, forcing a fit to the strong localization form [9], $R \propto \exp \left[\left(T_{0} / T\right)^{-1 / 2}\right]$, yields $T_{0}<20 \mathrm{mK}$. Such a low value for $T_{0}$ is not consistent 


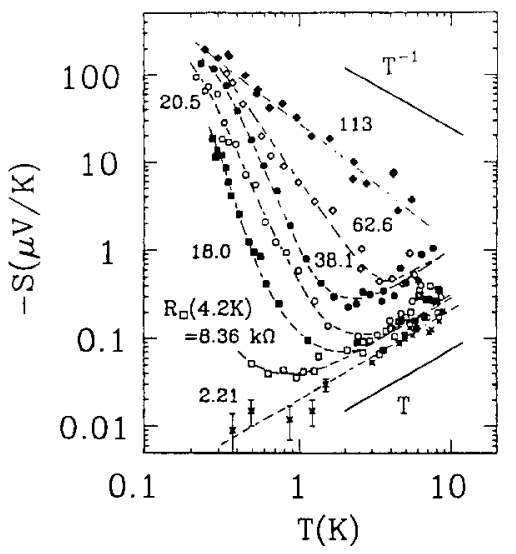

Fig. 4. Thermoelectric power versus temperature for the same film for which resistance data is shown in Fig. 3. Dashed lines are guides to the eye

with the condition $T \gg T_{0}$ for which this expression applies.

The thermoelectric power, $S$, for the same film is shown in Fig. 4. Similar thermopower data have been observed in three separate films, each oxidized in steps to span the $1<R<100 \mathrm{k} \Omega$. For disordered thin films, phonon drag effects are rendered negligible and we interpret the data as reflecting the carrier diffusion thermopower. The negative sign indicates a dominant contribution from electrons. The thermopower of metals is expected to decrease linearly with temperature, and this behavior is observed down to the lowest temperatures for $R(4.2 \mathrm{~K}) \ll 8 \mathrm{k} \Omega$. For $R(4.2 \mathrm{~K})>8 \mathrm{k} \Omega$ this $T$-linear dependence gives way to a dramatic transition where $S$ diverges with decreasing temperature to values that are several orders of magnitude larger than those for samples having low $R$. Note that the temperature at which the thermopower diverges increases with increasing oxidation and the divergence becomes less rapid, tending toward an $S \propto 1 / T$ dependence. The latter behavior is characteristic of a thermally activated process, as in semiconductors where the energy gap, $E_{g}$, gives rise to a lowtemperature $\left(k_{B} T<E_{g}\right)$ thermopower, $S \propto E_{g} / 2 k_{B} T$.

For the arrangement of grains in our films we estimate an average geometric capacitance on the order of $C=5 \times 10^{-16} \mathrm{~F}$, which corresponds to a charging energy $E_{c} / k_{B} \cong 2 \mathrm{~K}$. From the slope of the data for the highest level of oxidation we find an activation energy of $\sim 1 \mathrm{~K}$, which compares favorably with our estimate of $E_{c}$. This suggests that in the limit of high oxidation the charging energy is close to its geometric value with the thermopower taking on a simple activated form, $S \propto E_{c} / k_{B} T$. This $1 / T$ behavior is expected for mesoscopic junctions (Sect. 4) and for discontinuous metal films when transport is by thermally activated tunneling between islands. However, a faster than $1 / T$ divergence is not predicted by a simple model.

It is possible that the sharp upturn in the thermopower for intermediate oxidation is associated with the fact that the films form an array of coupled junctions rather than a single junction. During the time between tunneling events across an intergrain junction, there can be a redistribution of the charge onto neighboring grains. Such

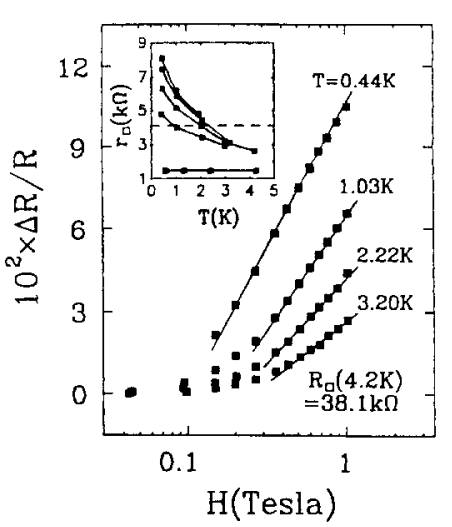

Fig. 5. Magnetoresistance at several temperatures for the oxidized film with $R(4.2 \mathrm{~K})=38.1 \mathrm{k} \Omega$. The inset shows $r$ versus $T$ for different oxidation levels as determined from the magnetoresistance data (see text). Values of $R(4.2 \mathrm{~K}$ ) are, from bottom to top (in $\mathrm{k} \Omega$ ): $1.47,8.36,18.0,38.1,62.6$. The dashed line is $r=\hbar e \approx 4.12 \mathrm{k} \Omega$

charge fluctuations will tend to increase the observed capacitance because the area probed by the electron is greater. The net result will be a decrease in the charging energy, yielding an effective value, $E_{c}^{\text {eff }}$. This process will be determined by the mobility of electrons on the network and thus should be more pronounced when the intergrain tunnel resistance $\left(R_{t}\right)$ is low. A similar picture might also be relevant for a single junction. For low $R_{t}$ the tunneling rate may be sufficiently high for an electron to tunnel back and forth several times during the interval in which it contributes to transport. It is possible that the mobility which determines the charge redistribution in our films is temperature dependent. This would imply a $T$-dependent $E_{c}^{\text {eff }}$, which may be pertinent to the thermopower data. These ideas require further theoretical support. We now discuss magnetoresistance measurements which suggest that the average intergrain tunnel resistance in the films increases with oxidation and with decreasing temperature.

For $R(4.2 \mathrm{~K})<8 \mathrm{k} \Omega$ these films exhibit the same weak localization magnetoresistance (MR) that is predicted and measured for homogeneous $\mathrm{Bi}$ films. The phase breaking length at $T=1 \mathrm{~K}$ is $L_{\phi} \simeq 1000 \AA$. For $H>H_{\phi} \equiv \phi_{0} / 4 \pi L_{\phi}^{2}\left(\phi_{0}\right.$ is the flux quantum) and in the presence of strong spin orbit scattering (antilocalization) we have

$\frac{\Delta R}{R}=\frac{R(H)-R(O)}{R(O)}=\frac{e^{2}}{4 \pi^{2} \hbar} r \ln H$,

where $r$ is the sheet resistance of a homogeneous film. The MR for $R(4.2 \mathrm{~K})=38.1 \mathrm{k} \Omega$, shown at several temperatures in Fig. 5, has a dependence that is qualitatively similar to weak localization, behaving as $\ln (H)$ in high fields and $H^{2}$ at low fields. However, fitting to (40) yields $r \ll R$. This is to be expected since $R$ reflects percolation effects whereas $r$ is a measure of the sheet resistance on the scale of the Landau orbit radius, $L_{H}$. This is the characteristic diffusion length which cuts off the coherent backscattering responsible for weak localization in a field $H>H_{\phi}$. In the logarithmic field regime this length is smaller than the average grain diameter for our films. The following arguments lead us to conclude that $r$ meas- 


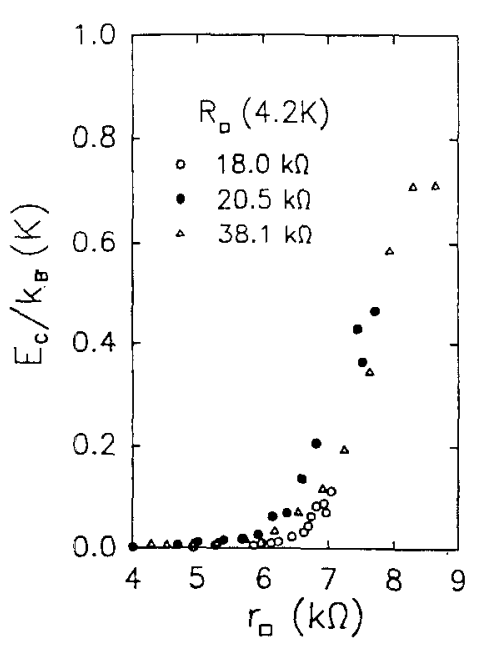

Fig. 6. The charging energy $E_{c}$, as determined from the thermopower data, plotted verus $r$ for three levels of oxidation (values of $R(4.2 \mathrm{~K}))$

ures the average intergrain tunnel resistance for highly oxidized films. Consider two points within a grain, one at the center and the other near the junction with a neighboring grain. The contribution at each such point to the $\ln (H)$ magnetoresistance is determined by summing the backscattering amplitudes associated with elastic scattering "trajectories" (and their time-reversed contours), each of which begins and ends at that point and have lengths less than $L_{H}$. For the point at the center of the grain all relevant trajectories are within the grain and hence the MR is the same as that for a homogeneous film with a sheet resistance equal to that of the weakly disordered grains (i.e. $<\mathrm{k} \Omega$ ). For the point near the junction we must consider trajectories which cross the junction and return. As long as these trajectories are statistically independent, the contribution to the MR will have the same form as for the center of the grain, but will be weighed by the intergrain tunneling resistance $R_{t}$. When the latter is much larger than the intragrain sheet resistance, as in the highly oxidized films, the overall prefactor of the total MR is predominantly determined by $R_{t}$.

The inset in Fig. 5 shows $r(T)$, as determined using (40), for different levels of oxidation. A most remarkable observation is that, for each level of oxidation, $r$ crosses $\hbar / e^{2}$ at approximately the same temperature below which the thermopower data deviate from metallic ( $T$-linear) behavior. This is consistent with our suggestion that $E_{c}^{\text {eff }}$ depends on temperature through $R_{t}(T)$. Extending this idea further, in Fig. 6 we relate the magnetoresistance and thermopower data by plotting $E_{c}$ versus $r$. We first extract the dependence of $E_{c}$ on temperature by assuming that the thermopower is given by $S \propto E_{c}^{\text {eff }} / k_{B} T$. We draw $1 / T$ curves through each of the $S(T)$ data points at temperatures below the crossover and $E_{c}^{\text {eff }}$ is then obtained from the slope. We next determine $E_{c}(r)$ by interpolating from the $r(T)$ data (Fig. 5, inset). The universal sharp transition in $E_{c}$ versus $r$ for both different oxidation and temperatures may reflect a fundamental interplay between Coulomb interaction effects and localization in granular metals. This poses a stimulating theoretical problem for future investigation.

\section{Conclusions}

We have demonstrated that the thermoelectric effects provide a very sensitive method to study the normalized DOS structure and the interaction of the mesoscopic junction with the "external field". In recent years the STM, and especially the CSTM (cryogenic STM), have been used for spectroscopic measurements (measurements of the DOS) in addition to topographic measurements. Wilkins et al. [10] have shown that charging effects (Coulomb blockade) due to the single charge transfer via oxide impurities may obscure the measurements of the DOS and lead to a wrong interpretation (e. g., they can lead to a gap-like structure in the DOS). The results presented here suggest a method to overcome these difficulties by the complementary (to the $I-V$ characteristic) measurement of the thermopower coefficient. Such measurements at relatively high temperatures (hence no charging) have been performed by William and Wickramasinghe [11] followed by the theoretical study of Stovneng and Lipavsky [12]. Leavens and Aers [13] presented a pioneering study of "Vacuum tunneling thermopower" with focus on the effect of the image potential.

In a forthcoming publication we show that the sensitivity can be further increased using two serially coupled junctions. It is also shown that the thermopower effects provide an excellent method to study resonant tunneling versus consecutive tunneling in serially connected tunnel barriers, as well as dephasing between tunneling events.

We have benefitted from conversations with Y. Gefen, R.C. Jaklevic, M. Frenk, A. Stern, and R. Wilkins. This research was partly supported by the Wolfson Foundation through the Israel Academy of Science, and a grant from the Ford Motor Company.

\section{References}

1. Other papers in this volume, Z. Phys. B - Condensed Matter 85, 319-468 (1991) and references therein

2. Amman, M., Ben-Jacob, E., Cohn, J.: Phys, Lett. A (submitted for publication)

3. Callen, H.B.: Phys. Rev. 73, 1349 (1948)

4. As was pointed out by Harrison (Harrison, W.A.: Phys. Rev. 123, 85 (1961)), for an ideal planar junction the tunneling matrix element is proportional to the inverse of the density of states. Here, when we refer to the normalized density of states, we refer to the energy dependent part of the density of states and the tunneling matrix element product. The normalized density of states is then proportional to the square root of the conductivity

5. Buttiker, M., Landauer, R.: Phys. Rev. Lett. 49, 1739 (1982)

6. Amman, M., Wilkins, R., Ben-Jacob, E., Maker, P.D., Jaklevic, R.C.: Phys. Rev. B43, 1146 (1991)

7. Cohn, J.L., Ben-Jacob, E., Uher, C.: Phys. Lett. A148, 110 (1990)

8. Lee, P.A., Ramakrishnan, T.V.: Rev. Mod. Phys. 57, 287 (1985)

9. Efros, A.L., Shklovskii, B.I.: J. Phys. C8, L49 (1975)

10. Wilkins, R., Amman, M., Ben-Jacob, E., Jaklevic, R.C.: Phys. Rev. B42, 8698 (1990)

11. Williams, C.C., Wickramasinghe, H.K.: Nature 344, 317 (1990)

12. Stovneng, J.A., Lipavsky, P.: Phys. Rev. B42, 9214 (1990)

13. Leavens, C.R., Aers, G.C.: Solid State Commun. 61, 289 (1987) 